\title{
An Update on Crossmatch Techniques in Transplantation
}

Kumar $\mathbf{A}^{1,2}$, Mohiuddin $\mathbf{A}^{2,3}$, Sharma $\mathbf{A}^{2,3}$, El Kosi $\mathbf{M}^{2,4}$ and Halawa $\mathbf{A}^{2,5^{*}}$

${ }^{1}$ Department of Nephrology, Zulekha Hospital, Dubai, UAE

${ }^{2}$ Institute of Clinical Science, School of Medicine, University of Liverpool, United Kingdom

${ }^{3}$ Department of Nephrology and Transplantation, Royal Liverpool University Hospital, United Kingdom

${ }^{4}$ Department of Nephrology, Doncaster Royal Infirmary, Doncaster, United Kingdom

${ }^{5}$ Department of Nephrology, Sheffield Kidney Institute, Sheffield, United Kingdom

"Corresponding author: Ahmed Halawa, Department of Nephrology, Sheffield Kidney Institute, Sheffield, United Kingdom, Tel: 00447787542128; Email: ahmed.halawa@sth.nhs.uk

Received date: Nov 02, 2017; Accepted date: Dec 23, 2017; Published date: Dec 29, 2017

Copyright: $\odot 2017$ Kumar A, et al. This is an open-access article distributed under the terms of the Creative Commons Attribution License, which permits unrestricted use, distribution, and reproduction in any medium, provided the original author and source are credited.

\section{Abstract}

Crossmatch techniques formulated nearly a half century ago remain the bedrock of transplantation science. While initial methods relied on complement dependent cytotoxicity. More recently additions to the transplant physician's immunologic armamentarium have arrived in both cell-based as well as solid phase assays. Flow cytometry is useful in previously sensitized patients while bead technology has revolutionised HLA antibody testing. The virtual crossmatch uses bead technology to detect specific recipient antibodies without requiring donor serum. We present a concise review of the techniques available, their basis and comparison between them so as to better understand their respective applications.
\end{abstract}

Keywords: Crossmatch; Renal transplant; Hyperacute rejection; Blood transfusion; Cytotoxicity

\section{Introduction}

The genesis of crossmatching was an attempt to identify those transplant recipients who had a higher likelihood of acute vascular rejections after receiving the graft from a given donor. This hyperacute rejection is the result of the presence preformed antibodies in the donor serum to one or many HLA (human leucocyte antigens). These are referred to as DSAs (donor specific antibodies).

DSAs are commonly generated by: (i) Blood transfusion (ii) Previous transplantation (iii) Pregnancy. One important implication of this is that wives often develop HLA antibodies against their husband's HLA in the course of conception. This may preclude the spouse as a donor in the event of renal failure. DSAs are conventionally identified with cell based assays, solid phase and more recently the virtual crossmatch. A basic understanding of the techniques used and the relative advantages and drawbacks of each allow better interpretation of results by the transplant physician.

\section{A Review of Crossmatch Techniques}

\section{Cell based assays}

CDC technique: Complement dependent cytotoxicity crossmatching was first developed in the 1960s. In 1969, Terasaki and Patel first observed that presence of donor specific antibodies in transplant recipiects were associated with a higher incidence of acute and hyperacute graft rejection [1]. The significance of CDC lies in its ability to determine the presence of donor specific anti- HLA antibodies in the serum of the recipient, thus helping prognosticate graft survival after transplant. The technique involved isolating donor lymphocytes (cadaveric/ living). The B and $\mathrm{T}$ cells are separately tested against serum from the recipient. Humeral immunological response is mediated though activation of the complement system by the classical pathway. To demonstrate the effects of this cascade, complement is added to the mixed recipient serum and donor lymphocytes and cell lysis of the lymphocytes is observed. The CDC crossmatch technique has been shown in Figure 1 given below.

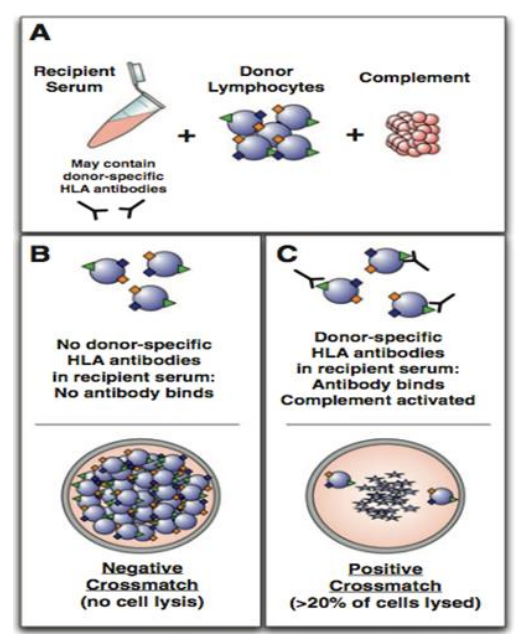

Figure 1: CDC crossmatch (A) Serum from the recipient is added to donor lymphocytes ( $\mathrm{T}$ or $\mathrm{B}$ ) in the presence of complement. (B) Negative test when donor-specific anti-HLA antibodies are absent and complement activation does not occur. (C) Positive test when donor-specific antibodies bind to lymphocytes, activate complement and cause cell lysis. 
The result is represented in terms of the percentage of lymphocytes in the cell panel which has undergone lysis as a result of complement activation or panel-reactive antibody (\% PRA) [2]. Twenty percent is usually taken as the minimum cut-off for a positive result thus functioning as a qualitative and a semi-quantitative estimate of the strength of the reaction. The other method that can help quantify the strength of this reaction is the "titred crossmatch" method in which a crossmatch of the donor lymphocytes is performed using serial doubling dilutions of the recipient's serum. Thus, higher the dilution required to give a negative result, greater the strength of the immune reaction. This also helps determine the need for a desensitization protocol for the recipient prior to transplant. Addition of anti-human immunoglobulin (AHG) helps increase the sensitivity of CDC as multiple AHGs bind to a single donor specific antibody, amplifying its complement activation response at smaller titres.

Limitations of CDC include false positive and negative results. The former is often a result of autoantibodies in the recipient serum. This can be overcome by addition of Dithiothreitol (DTT) which helps prevent predominantly IgM autoantibody mediated complement activation and allows only IgG (donor specific antibodies) [3]. False negative reactions may occur when DSA levels are too low to result in activation of the complement cascade or if the antibodies are of the type that does not cause complement activation [4]. Antibody levels of the recipient may vary with time due to new antigen exposures and immunological sensitization that may occur after antigen exposure in the form of blood transfusions, pregnancy, etc [5]. This emphasized the importance of matching the most recently drawn recipient serum sample with the donor lymphocytes, especially in the context of patients on a donor transplant list for cadaveric transplant.

HLA (human leucocyte antigen) are encoded for by genes on chromosome 6 that at inherited en-bloc. Class I antigens (HLA-A, B, C) are expressed on all nucleated cells while class II antigens HLA-DR, DP, DQ) are expressed on antigen presenting cells like macrophages and dendritic cells. Vascular endothelial cells of the transplant graft express both these antigens, hence antibody responses to them are of importance in predicting acute antibody mediated rejection. $\mathrm{T}$ cells express only class I antigens while B cells express both. Hence, a positive $\mathrm{T}$ cell and $\mathrm{B}$ cells cross match would indicate presence of antibodies to HLA type I and II antigens while a positive B cell cross match could indicate either (i) DSAs to type II antigens alone or (ii) Low levels of DSAs to type 1 antigens. A positive $T$ cell crossmatch alone is usually due to technical error.

Outcomes in positive T-cell crossmatch results: After ruling out the presence of autoantibodies, a renal transplant performed when the recipient has a positive $\mathrm{T}$ cell cross match results in poor outcome and unacceptably high incidence of acute graft rejection as shown in studies by Terasaki et al. Later, Stegall and colleagues, in a study done on $\mathrm{T}$ cell crossmatch positive recipients concluded that multiple PP treatments lead to reproducible desensitization and low acute and hyperacute graft rejection rates due to humoral immune response [6]. However, a persistent positive $\mathrm{T}$ cell crossmatch result post desensitization is according to current guidelines, an absolute contraindication to renal transplant.

Outcomes in positive B-cell crossmatch results: A positive B cell crossmatch is not as consistently associated with humoral rejection as positive $\mathrm{T}$ cell crossmatches. Although a negative B cell crossmatch is associated with better outcomes, the test's high false positive rate makes its significance uncertain. This can be overcome by concurrent Luminex testing for DSAs which increases its specificity. In case of true positive B cell crossmatches, the significance of anti-class II HLA antibodies is of less (albeit not nil) significance in acute/ hyperacute rejection and still warrant desensitization of the recipient prior to transplant [7].

(2) Flow cytometry: Flow cytometry for crossmatching was a technique first described in the context of pre transplant in the early eighties. Here, the donor lymphocytes are mixed with the recipient's serum. These bind to the donor specific antibodes and are quantified by detectors in the impedence flow cytometer [8]. Flow cytometry crossmatch technique has been shown in Figure 2. Two methods of quantification can be used. They are given below:

(i) Measurement of the fluorescence intensity as a ratio of the control (channel shifts).

(ii) Serial dilutions of the recipient's serum are made to react with donor lymphocytes and the minimum dilution which yields a negative result gives a measurable estimate.

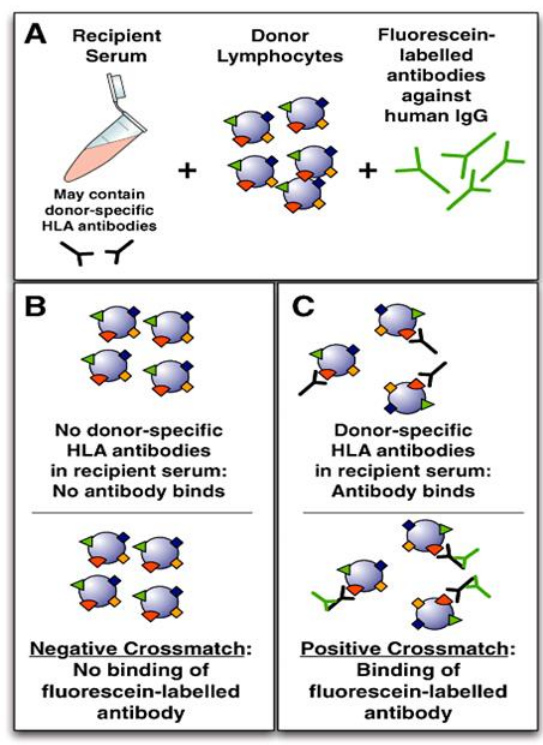

Figure 2: Flow cytometry crossmatch (A) Serum from the recipient is added to donor lymphocytes ( $\mathrm{T}$ or $\mathrm{B}$ ) in the presence of anti-IgG fluorescein-labelled antibodies. (B) Negative test when donorspecific antibodies are absent and binding does not occur. (C) Positive test when donor-specific antibodies bind to lymphocytes that are detected by flow cytometry once the anti-IgG fluoresceinlabelled antibodies tag the lymphocytes.

\section{Interpretation of flow crossmatch results}

In the light of a negative CDC result, a positive flow cytometry could be interpreted as indicating the presence of any of the following

\section{Non-complement fixing antibodies}

\section{Non-HLA antibodies}

Low-level antibodies

Hence, the value of this test's sensitivity lies in using it for cross matching of sensitized patients who have an inherently higher risk of acute graft rejection to determine transplant feasibility or need for desensitization protocols prior to transplant [9]. A large retrospective 
study based on data from the organ procurement and transplant network registry showed that CDC crossmatch negative patients who had positive T-cell flow cytometry results had significantly poorer absolute 5 year graft survival rates that those who were both CDC and flow cytometry crossmatch negative [10].

\section{Solid phase antibody detection assays}

Enzyme-linked immunosorbent assay (ELISA): The initial application of ELISA was to detect HLA in both bound and free forms but its current utility is for the detection of HLA antibodies in serum. The modified assay uses HLA glycoprotein immobilized into mictrotiter wells. The recipient serum is added and specific antibodies bind the epitopes available. A wash is performed and anti-IgG with a passenger reported molecule (alkaline phosphatase) is added that combines with the anti-HLA antibody. After further washing to remove unbound antibody, a substrate is added which after dephosphorylation by the reporter molecule undergoes a color change.

This method while effective in detecting sensitization in transplant candidates has been replaced by bead technology [11].

Bead technology (Luminex): HLA antibody testing was revolutionised by the introduction of beads labeled with flourescein in the nineties.

Generally beads are impregnated with different ratios of two fluorochromes resulting in a signal that is unique to the specific bead. Each bead may have one or more HLA molecule types incorporated. The basic steps involve first, incubation of recipient serum with the beads. HLA antibodies of the serum will react with HLA antigens on the bead. The beads are washed and incubated with a second antibody, usually anti-human IgG labeled with phycoerythrin.

\section{Levels of testing}

(i) Screening: Beads are incorporated with a many molecules, both class I and II derived from cell lines. (ii) Testing against a set of alleles from an individual genome: Beads have HLA molecules from a single cell line with two molecules for each HLA locus. These results can be expressed as PRA. (iii) Single antigen beads: These beads have a single type of HLA antigen molecules bound to them. The result is that a conglomerate of heterogenous antibodies can be tested and specific HLA antigens can be identified [11].

\section{Quantitation of the result}

This is achieved by two methods: (i) Flow cytometry: Measure the channel shift that is associated with binding of antibody [12]. (ii) Luminex method: Two lasers are used to excite the fluorochrome of the bead and the phycoerythrin bound to the antibody. The degree of fluorescence is calculated as a mean fluorescence intensity (MFI) [13].

\section{Interpreting flow crossmatch studies}

The main significance of a positive test if the corresponding CDC crossmatch was negative. In this scenario, the reason for the positive match could be due to a low level antibody, a non-HLA antibody or a non-complement binding antibody. Another issue is that the cut-off values are not uniform cross laboratories. Low cut offs would increase sensitivity while affecting specificity and vice-versa [14]. Single antigen beads are employed in allowing semiquantitative evaluation of HLA antibodies in order to predict pretransplant risk as well as for donor specific antibody monitoring after transplant [15].

\section{The virtual crossmatch}

This technique is based on the comparison of the anti-HLA antibodies of the recipient to the donor HLA antigens using bead technology. This method predicts the eventual crossmatch and can assist in rapid identification of a suitable donor [16]. Multiple synthetic microspheres are each given a single HLA antigen coating and incubated with recipient serum. Virtual crossmatch techniques has been shown in Figure 3.

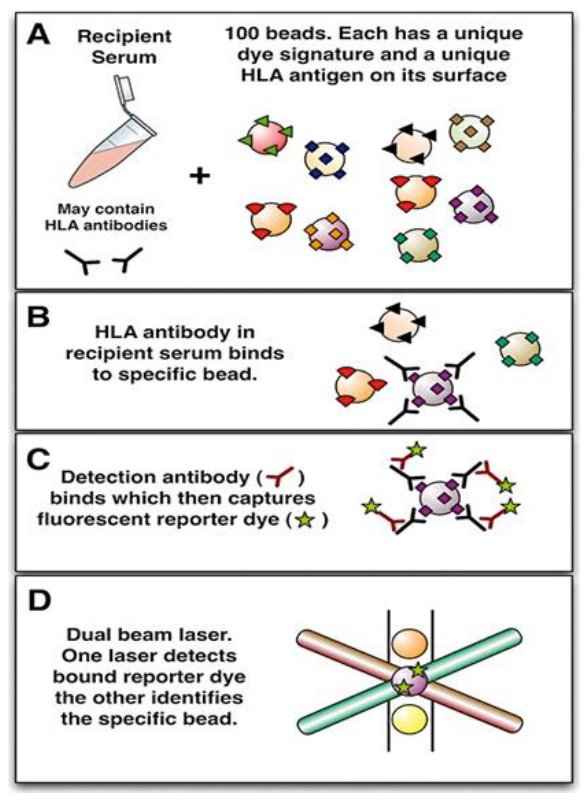

Figure 3: Virtual crossmatch (A) Serum from the recipient is added to synthetic beads with either a set of antigens or a single antigen, each bead can be identified by an independent dye signature. (B) Anti-HLA antibodies if present will bind to the specific bead. (C) A detector antibody will then bind and sequester a reporter dye. (D) Beads can be checked for the reporter dye using a laser beam, this builds a profile of the antibodies present in the recipient that can be compared with the HLA construct of a potential donor thus predicting the result of crossmatch.

Alternatively a microsphere or bead may be coated with multiple HLA antigens - this iteration of the test allows for more efficient screening. HLA antibodies bind specifically with the bead and are detected by isotype specific antibodies using flow cytometry. This method allows for the sensitivity of flow crossmatch combined with identifying specific antibody. Johnson et al. found that the final transplant decision could be taken on the basis of the virtual crossmatch irrespective of the flow cytometry without significant difference in the clinical outcome in those who were flow cytometry positive but virtual crossmatch negative [17]. A recent study on pancreas transplants showed a reduced cold ischemia time when virtual crossmatch was used in lieu of flow cytometry [18]. This decrease in ischemia time was achieved without increasing rejection or affecting graft survival. A corollary would be that this technique would be valuable for programs sharing organs over significant distances [18]. 


\section{Comparison of Techniques}

\section{Complement dependent cytotoxicity}

Advantages: It represents a functional test that involves cells and serum containing antibodies. A basic and durable test still used as the foundation of crossmatch due to availability, cost and simplicity.

It has played a key role in preventing hyperacute rejection as the science of transplant immunology progressed.

It detects only complement binding antibodies.

Drawbacks: It is less sensitive than other newer assays.

It detects $\operatorname{IgM}$ and IgG antibodies simultaneously, autoantibodies and non-HLA antibodies against antigens that are irrelevant as far as the transplant is concerned.

\section{Flow cytometry}

Advantages: More sensitive than CDC crossmatch. It can detect lesser levels of IgG HLA antibodies and has less inter-observer variability [19].

Drawbacks: Slow turnaround times meant this technique was suitable for living donors scenarios rather than for cadaveric donors.

\section{Solid phase assays (ELISA and bead technology)}

Advantages: They are the most sensitive of all the techniques for detecting donor specific HLA antibodies.

Enhanced sensitivity has allowed improved rates of success in retransplants where detection of pre-sensitization from previous grafts allow the avoidance of those HLA markers on subsequent grafts [20].

They allow the identification of all HLA alleles for which the recipient harbours antibodies.

They avoid false positives of antibody binding to non-HLA antigens and eliminates confusion regarding the class of HLA that are detected (CDC matching has an overlap between $\mathrm{T}$ and $\mathrm{B}$ cell matching, $\mathrm{T}$ with Class I and B with both class I and II).

Drawbacks: The interpretation of a positive assay in the presence of negative CDC/flow crossmatch is ambiguous. The relevance of low level antibodies to low significance antigens is debatable.

Single antigen bead assays detect both complement-fixing and noncomplement-fixing HLA antibodies. On the basis of these results, prospective recipient may be denied a graft without established clinical significance. 3. Other limitations include interference by $\operatorname{IgM}$, incomplete library of antigens in the bead sets, variability of HLA density on the beads.

\section{Conclusion}

Complement dependent cytotoxicity remains the mainstay of pretransplant screening for HLA specific antibodies. Newer methods have helped significantly increase the sensitivity and specificity of detecting antibodies of significance in acute humoral graft rejection in the recipient when interpreted in the light of the patient's clinical picture and CDC crossmatch testing whilst accounting for inter-lab variations and technical errors. Flow crossmatch and Luminex bead assays allows the identification of lower titre, possibly clinically significant anti HLA antibodies. At present, we have an incomplete understanding of low level donor specific, non HLA and non-complement fixing antibodies in terms of their significance in solid organ transplant. The combination of these various assays allow us to exclude insignificant antibodies form risk assessment while allowing better prognostication and preparation when more significant antibodies are identified that can potentially complicate though not preclude transplant.

\section{Acknowledgement}

We thank Dr. William R Mulley for affording us the reproduction of descriptive images in this article.

\section{References}

1. Patel R, Terasaki PI (1969) Significance of the positive crossmatch test in kidney transplantation. N Engl J Med 280: 735-739.

2. Gebel HM, Bray RA (2014) HLA antibody detection with solid phase assays: Great expectations or expectations too great? Am J Transplant 14: 1964-1975.

3. Khodadadi L, Adib M, Pourazar A (2006) Immunoglobulin class (IgG, IgM) determination by dithiothreitol in sensitized kidney transplant candidates. Transplant Proc 38: 2813-2815.

4. Mulley WR, Kanellis J (2011) Understanding crossmatch testing in organ transplantation: A case-based guide for the general nephrologist. Nephrology (Carlton) 16: 125-133.

5. Gebel HM, Bray RA, Nickerson P (2003) Pre-transplant assessment of donor-reactive, HLA-specific antibodies in renal transplantation: Contraindication vs. risk. Am J Transplant 3: 1488-1500.

6. Stegall MD, Gloor J, Winters JL, Moore SB, Degoey S (2006) A comparison of plasmapheresis versus high-dose IVIG desensitization in renal allograft recipients with high levels of donor specific alloantibody. Am J Transplant 6: 346-351.

7. Pollinger HS, Stegall MD, Gloor JM, Moore SB, Degoey SR, et al. (2007) Kidney transplantation in patients with antibodies against donor HLA class II. Am J Transplant 7: 857-863.

8. Downing J (2012) The lymphocyte crossmatch by flow cytometry for kidney transplantation. Methods Mol Biol 882: 379-390.

9. Karpinski M, Rush D, Jeffery J, Exner M, Regele H, et al. (2001) Flow cytometric crossmatching in primary renal transplant recipients with a negative anti-human globulin enhanced cytotoxicity crossmatch. J Am Soc Nephrol 12: 2807-2814.

10. Graff RJ, Buchanan PM, Dzebisashvili N, Schnitzler MA, Tuttle-Newhall J, et al. (2010) The clinical importance of flow cytometry crossmatch in the context of CDC crossmatch results. Transplant Proc 42: 3471-3474.

11. Tait BD (2016) Detection of HLA antibodies in organ transplant recipients-triumphs and challenges of the solid phase bead assay. Front Immunol 7: 570 .

12. Rebibou JM, Chabod J, Bittencourt MC, Thévenin C, Chalopin JM, et al. (2000) Flow-PRA evaluation for antibody screening in patients awaiting kidney transplantation. Transplant Proc 32: 2745-2746.

13. Colombo MB, Haworth SE, Poli F, Nocco A, Puglisi G, et al. (2007) Luminex technology for anti-HLA antibody screening: Evaluation of performance and of impact on laboratory routine. Cytometry B Clin Cytom 72: 465-471.

14. Tait BD, Hudson F, Cantwell L, Brewin G, Holdsworth R, et al. (2009) Review article: Luminex technology for HLA antibody detection in organ transplantation. Nephrology (Carlton) 14: 247-254.

15. Ellis TM (2013) Interpretation of HLA single antigen bead assays. Transplant Rev (Orlando) 27: 108-111.

16. Morris GP, Phelan DL, Jendrisak MD, Mohanakumar T (2010) Virtual crossmatch by identification of donor-specific anti-human leukocyte antigen antibodies by solid-phase immunoassay: A 30 month analysis in living donor kidney transplantation. Hum Immunol 71: 268-273. 
Citation: Kumar A, Mohiuddin A, Sharma A, El Kosi M, Halawa A (2017) An Update on Crossmatch Techniques in Transplantation. J Kidney 3: 160. doi:10.4172/2472-1220.1000160

Page 5 of 5

17. Johnson CP, Schiller JJ, Zhu YR, Hariharan S, Roza AM, et al. (2016) Renal transplantation with final allocation based on the virtual crossmatch. Am J Transplant 16: 1503-1515.

18. Eby BC, Redfield RR, Ellis TM, Leverson GE, Schenian AR, et al. (2016) Virtual HLA crossmatching as a means to safely expedite transplantation of imported pancreata. Transplantation 100: 1103-1110.
19. Phanish MK (2016) Immunological risk assessment and human leukocyte antigen antibody testing in kidney transplantation. Indian J Nephrol 26: 80-85.

20. Picascia A, Infante T, Napoli C (2012) Luminex and antibody detection in kidney transplantation. Clin Exp Nephrol 16: 373-381. 